\title{
High-resolution spectroscopy, crystal-field calculations, and quadrupole helix chirality of $\mathrm{DyFe}_{3}\left(\mathrm{BO}_{3}\right)_{4}$
}

\author{
M.N. Popova ${ }^{1, *}$, B.Z. Malkin², T.N. Stanislavchuk ${ }^{3}$, E.P. Chukalina ${ }^{1}$, K.N. Boldyrev ${ }^{1}$, and \\ I.A. Gudim ${ }^{4}$ \\ ${ }^{1}$ Institute for Spectroscopy RAS, 142190 Troitsk, Moscow, Russia \\ ${ }^{2}$ Kazan Federal University, 420008 Kazan, Russia \\ ${ }^{3}$ Department of Physics, New Jersey Institute of Technology, NJ 07102 Newyark, USA \\ ${ }^{4}$ Kirensky Institute of Physics, Siberian Branch of RAS, 660036 Krasnoyarsk, Russia
}

\begin{abstract}
High-resolution polarized transmission spectra of $\mathrm{DyFe}_{3}\left(\mathrm{BO}_{3}\right)_{4}$ single crystals were investigated in broad spectral $\left(10-23000 \mathrm{~cm}^{-1}\right)$ and temperature $(3.5-300 \mathrm{~K})$ ranges. Energies of the dysprosium levels in the paramagnetic and antiferromagnetic phases were determined. On the basis of these data and preliminary calculations in the frameworks of the exchange-charge model, we determined the crystal-field and $\mathrm{Dy}-\mathrm{Fe}$ exchange interaction parameters of the $\mathrm{Dy}^{3+}$ ions at sites with the point $C_{2}$ symmetry corresponding to the enantiomorphic $P 3_{1} 21$ and $P 3_{2} 21$ space groups. The values of electronic quadrupole moments of the $\mathrm{Dy}^{3+}$ ions were calculated, which enabled us to interpret results of the work [Usui et al., Nature Mater. 13, 611 (2014)] on the observation of domains of different quadrupole chirality in $\mathrm{DyFe}_{3}\left(\mathrm{BO}_{3}\right)_{4}$.
\end{abstract}

The crystals $\mathrm{DyFe}_{3}\left(\mathrm{BO}_{3}\right)_{4}$ belong to the family of multiferroic rare-earth iron borates that crystallize in the trigonal $R 32$ structure of the natural mineral huntite. With decreasing the temperature to $T_{\mathrm{S}}=285 \mathrm{~K}$, DyFe ${ }_{3}\left(\mathrm{BO}_{3}\right)_{4}$ undergoes a structural phase transition from the $R 32$ phase to the enantiomorphic space-group pair $P 3_{1} 21$ and $P 3_{2} 21$. It has been shown recently by resonant $\mathrm{x}$-ray diffraction that, below $T_{\mathrm{S}}$, single-crystal samples of $\mathrm{DyFe}{ }_{3}\left(\mathrm{BO}_{3}\right)_{4}$ contain macroscopic domains which differ by right-handed $\left(P 3_{1} 21\right)$ or left-handed $\left(P 3_{2} 21\right)$ helical structures of electronic quadrupole moments of the $\mathrm{Dy}^{3+}$ ions [1]. Quadrupole moments at the Dy sites are induced by a low-symmetry $C_{2}$ component of the crystal field that appears below $T_{\mathrm{S}}$ and distorts the electronic density distribution in the $\mathrm{Dy}^{3+}$ ions. It is possible to calculate values of these moments on the base of the crystal-field $(\mathrm{CF})$ parameters. A limited available information on the $\mathrm{CF}$ levels of $\mathrm{Dy}^{3+}$ ions in $\mathrm{DyFe}_{3}\left(\mathrm{BO}_{3}\right)_{4}$ prevents from doing $\mathrm{CF}$ calculations in the frame of the $C_{2}$ symmetry CF Hamiltonian with 15 parameters.

We have measured temperature-dependent transmission spectra of $\mathrm{DyFe}_{3}\left(\mathrm{BO}_{3}\right)_{4}$ single crystals corresponding to optical transitions from the ground multiplet to all the excited multiplets up to about $22200 \mathrm{~cm}^{-1}$ and to the far-infrared transitions within the ground multiplet. Almost all of the spectral lines observed in the absorption spectra of both the

*Corresponding author: popova@isan.troitsk.ru 
paramagnetic and antiferromagnetic $\left(\mathrm{T}<T_{\mathrm{N}}=39 \mathrm{~K}\right)$ phases of $\mathrm{DyFe}_{3}\left(\mathrm{BO}_{3}\right)_{4}$ were unambiguously identified and the corresponding energies of excitations of the $\mathrm{Dy}^{3+}$ ions were determined. This dataset formed a basis for the CF calculations and modeling of exchange interactions. Here, we confine ourselves with the paramagnetic phase.

The spectrum of a $\mathrm{Dy}^{3+}$ ion in a dielectric crystal can be described using the Hamiltonian $H=H_{\mathrm{FI}}+H_{\mathrm{CF}}$, where $H_{\mathrm{FI}}$ is the free-ion Hamiltonian but $H_{\mathrm{CF}}=\sum_{p, q} C_{q}^{p} B_{q}^{p}$ is the CF Hamiltonian determined by 15 independent CF parameters $B_{q}^{p}\left(C_{q}^{p}\right.$ are the spherical tensor operators of rank $p$ ). The initial values of the CF parameters were calculated in the framework of the exchange-charge model [2]. Then, the energies of transitions between the levels of the $\mathrm{Dy}^{3+}$ ions, obtained from the numerical diagonalization of the Hamiltonian $H$ operating in the total space of 2002 states of the electronic $4 f^{\ominus}$ configuration, were compared with the measured optical spectra of $\mathrm{DyFe}_{3}\left(\mathrm{BO}_{3}\right)_{4}$ in the paramagnetic phase, and the initial CF parameters were varied to fit the experimental data. Values of the six CF parameters $\left(B_{0}^{2}=404, \quad B_{0}^{4}=-1192, \quad B_{-3}^{4}=B_{3}^{4}=554 i, \quad B_{0}^{6}=328, \quad B_{-3}^{6}=B_{3}^{6}=70 i, \quad B_{6}^{6}=B_{-6}^{6}=232 \mathrm{~cm}^{-1}\right)$ which determine the dominant trigonal $\mathrm{CF}$ component change monotonously along the series of RE iron borates. The remaining nine parameters of the CF component of the $C_{2}$ symmetry $\left(B_{-1}^{2}=B_{1}^{2}=58.4 i, \quad B_{2}^{2}=B_{-2}^{2}=69.4, \quad B_{-1}^{4}=B_{1}^{4}=-49.2 i, \quad B_{2}^{4}=B_{-2}^{4}=101.2, \quad B_{4}^{4}=B_{-4}^{4}=15.9\right.$, $\left.B_{-1}^{6}=B_{1}^{6}=-7.4 i, \quad B_{2}^{6}=B_{-2}^{6}=-14, \quad B_{4}^{6}=B_{-4}^{6}=31.4, \quad B_{-5}^{6}=B_{5}^{6}=-79 i\right)$ are found with lower accuracy because they little influence the multiplet splittings. However, just this $C F$ component determines some specific properties of RE iron borates in the $P 3_{1} 21$ (or $P 3_{2} 21$ ) phase, in particular, the anisotropy of the magnetic spectroscopic factors of Kramers doublets of the $\mathrm{Dy}^{3+}$ ions in the $a b$-plane and the quadrupole helix chirality in $\mathrm{DyFe}_{3}\left(\mathrm{BO}_{3}\right)_{4}[1]$.

The low-symmetry $C_{2}$-component of the crystal field distorts the electronic density distribution in the $\mathrm{Dy}^{3+}$ ions and induces non-zero non-axial components of the electronic quadrupole moment at the dysprosium sites. The quadrupole helix chirality appears due to rotations of the local $C_{2}$ symmetry axis by $2 \pi / 3$ and $4 \pi / 3$ at the $3 a$ dysprosium sites shifted relative one another along the $c$-axis by $c / 3$ and $2 c / 3$, respectively. The results of calculations of the average values of the quadrupole moment components $<Q>=\operatorname{Tr}\left[Q \exp \left(-H / k_{\mathrm{B}} T\right)\right] / \operatorname{Tr}\left[\exp \left(-H / k_{\mathrm{B}} T\right)\right]$ at different temperatures $T\left(k_{\mathrm{B}}\right.$ is the Boltzman constant) using the set of the $\mathrm{CF}$ parameters given above agree satisfactorily with the experimental data presented in Ref. [1] (see Table 1) and confirm supposition [1] that changes of $\left\langle Q_{x^{2}-y^{2}}>\right.$ and $\left\langle Q_{y z}\right\rangle$ with temperature are mainly induced by redistribution of populations of $\mathrm{CF}$ sublevels of the $\mathrm{Dy}^{3+}$ ion ground multiplet.

Table 1. Quadrupole moments (arbitrary units) of $\mathrm{Dy}^{3+}$ in the $P 3_{12} 21$ phase of $\mathrm{DyFe}_{3}\left(\mathrm{BO}_{3}\right)_{4}$.

\begin{tabular}{ccccc}
\hline \multirow{2}{*}{ Temperature } & \multicolumn{2}{c}{$\left\langle Q_{x^{2}-y^{2}}\right\rangle$} & \multicolumn{2}{c}{$\left\langle Q_{y z}\right\rangle /\left\langle Q_{x^{2}-y^{2}}\right\rangle$} \\
\cline { 2 - 5 } & Measured [1] & Computed & Measured [1] & Computed \\
\hline $50 \mathrm{~K}$ & -1.22 & -1.18 & -1.23 & -1.415 \\
$100 \mathrm{~K}$ & -0.906 & -0.910 & -1.20 & -1.202 \\
$150 \mathrm{~K}$ & -0.678 & -0.695 & -1.10 & -1.096 \\
$200 \mathrm{~K}$ & -0.50 & -0.548 & -0.90 & -1.031 \\
\hline
\end{tabular}

This paper was supported by the Russian Science Foundation (14-12-01033).

\section{References}

1. T. Usui et al., Nature Materials 13, 611 (2014)

2. B.Z. Malkin, Ion-phonon interactions, Spectroscopic Properties of Rare Earths in Optical Materials (Springer, Berlin, 2005) 